Part II

\title{
THE SUN
}




\title{
LES SOURCES D'EMISSIONS RADIOELECTRIQUES DU SOLEIL
}

\section{INTRODUCTORY LECTURE by}

\author{
J. F. DENISSE \\ Observatoire de Meudon, Meudon (Seine-et-Oise), France
}

Si les émissions radioélectriques du soleil sont dépourvues de la richesse d'informations apportée par les spectres optiques, elles se révèlent, par contre, particulièrement bien adaptées à l'étude d'une très grande variété de perturbations solaires, dont bien souvent les observations optiques n'avaient même pas permis de soupçonner l'existence, et ceci pour plusieurs raisons:

1. Ces émissions, souvent transitoires, intéressent particulièrement la couronne, ou la haute chromosphère, où les observations optiques sont discontinues et difficiles en raison de l'éblouissement par le disque photosphérique qui n'existe pas en radio.

2. Certaines ne sont pas d'origine thermique: le plasma coronal possède diverses possibilités de rayonnement (oscillations de plasma, rayonnement synchrotron ou Čerenkov) plus aisément ou seulement perceptible dans le domaine radioélectrique.

Les sources solaires d'émissions radioélectriques sont multiples et se distinguent essentiellement par leur stabilité plus ou moins grande et la nature de leur rayonnement; pour le moment on ne possède pas beaucoup d'indications sur leurs dimensions, encore moins sur leur structure.

\section{SOLEIL CALME ET PROPAGATION DANS LA COURONNE}

L'atmosphère du soleil en dehors de toute perturbation rayonne dans le spectre hertzien le rayonnement free-free de l'hydrogène et de l'hélium ionisé. La fonction source dépend essentiellement de la densité $N_{e}$ des électrons et de leur température d'agitation $T_{e}$, et les observations effectuées sur ondes décimétriques et plus courtes apportent un contrôle utile aux modèles d'atmosphère déduits des données optiques (C. de Jager, paper 15, R. G. Athay, 16).

Toutefois, quand on confronte ces modeles d'atmosphère au rayonnement du soleil sur ondes métriques, on doit tenir compte que l'indice de réfraction du milieu coronal, qui dépend de $N_{e}$ et du champ magnétique $H$ dont on connaît peu de chose, peut être différent de l'unité. L'atmosphère solaire, surtout sur ondes longues, est partiellement réfléchissante; en présence d'un champ magnétique, elle devient en outre biréfringente; une onde de fréquence $f$ se décompose en deux composantes de polarisations différentes: l'une, l'onde ordinaire se propage pratiquement indépendamment du champ magnétique 
avec un indice

$$
n_{o}=\sqrt{1-\frac{f_{c}^{2}}{f^{2}}}
$$

l'autre, l'onde extraordinaire avec un indice

$$
n_{e}=\sqrt{1-\frac{f_{c}^{2}}{f\left(f-f_{B}\right)}} .
$$

La fréquence critique $f_{0}$ ne dépend que de $N_{e}$; la fréquence gyromagnétique $f_{H}$ ne dépend que de $H$. Quand on exprime $N_{e}$ en $\mathrm{cm}^{-3}, f_{c}$ et $f_{H}$ en $\mathrm{Mc} / \mathrm{s}$, et $H$ en gauss on a:

$$
f_{c}^{2}=0.81 \times 10^{-4} N_{e} \text { et } f_{B}=2.8 H .
$$

Les formules précédentes montrent qu'une onde de fréquence $f$ ne peut se propager dans l'atmosphère solaire qu'à une altitude suffisamment élevée pour que l'un des indices $n_{0}$ et $n_{e}$ soit réel; c'est-à-dire, que l'une au moins des conditions

$$
f \geq f_{c} \text { ou } f \geq \frac{1}{2}\left(f_{H}+\sqrt{\left.\overline{f_{H}^{2}+4 f_{c}^{2}}\right)}\right.
$$

soit satisfaite. Les valeurs $f_{c}$ et $f_{e}$, qui correspondent à une altitude donnée pour une atmosphére solaire standard et le champ magnétique d'une tache typique, sont indiquées dans le Tableau I.

\begin{tabular}{crrrrr}
\multicolumn{5}{c}{ TABLEAU I } \\
$r$ & 1.125 & 1.25 & 1.50 & 1.75 & 2 \\
$f_{c}(\mathrm{Mc} / \mathrm{s})$ & 117 & 75 & 40 & 24 & 17 \\
$f_{H}(\mathrm{Mc} / \mathrm{s})$ & 1700 & 330 & 40 & 25 & 5 \\
$f_{e}(\mathrm{Mc} / \mathrm{s})$ & 1710 & 355 & 62 & 41 & 20
\end{tabular}

Le fait qu'un rayonnement de fréquence $f$ émis par le soleil provient certainement d'une altitude au moins égale à celle qui correspond à $f=f_{\mathrm{c}}$ joue un rôle fondamental dans l'interprétation des différents types d'émissions solaires.

Il est possible que des phénomènes de diffusion dus aux irrégularités de la couronne solaire soient à considérer également pour l'interprétation des données radioélectriques. Mais jusqu'à présent toutes les données que l'on possède sur ces effets reposent sur l'éclipse de la nébuleuse du Crabe par l'atmosphère solaire qui intéresse seulement des régions de la couronne lointaine $(r>5)$; les mesures n'ont été faites qu'en ondes métriques pour lesquelles l'élargissement de l'image de la nébuleuse atteint une vingtaine de minutes (A. Hewish, 52, V. V. Vitkevich, 54, E. J. Blum et A. Boischot, 55).

\section{LES CONDENSATIONS RADIOELECTRIQUES}

Ces condensations radio sont responsables de la " composante lentement variable" du rayonnement solaire (slowly varying component). Ces sources d'émissions paraissent immobiles dans l'atmosphère solaire et leur rayonnement est remarquablement stable. Elles sont situées au-dessus des plages 
faculaires et leur altitude est relativement basse $(<100,000 \mathrm{~km})$. Leur durée de vie moyenne est intermédiaire entre celle des taches et celle des plages faculaires. Le spectre de leur rayonnement couvre tout le domaine des ondes centimétriques et décimétriques (W. N. Christiansen et D. S. Mathewson, 19; B. Vauquois, 26; J. Firor, 24; M. Gutmann et J. L. Steinberg, 21; V. V. Vitkevich, A. D. Kuz'min, V. A. Udal'tsov, et A. E. Salomonovich, 23; V. N. Ikhsanova, 30). On observe aussi des condensations sur ondes métriques, mais il n'est pas sûr qu'elles soient de même nature (A. Boischot et P. Simon, 25).

Il semble que l'on puisse considérer deux types de condensations:

1. Les unes, qu'on observe le plus souvent, sont relativement larges et peu intenses, leur rayonnement n'est pas polarisé. Les diamètres de ces condensations diffuses sont comparables aux diamètres des plages faculaires.

2. Les autres sont étroites et brillantes, leur diamètre, inférieur à la minute, est comparable à celui des taches et leur rayonnement est fortement polarisé circulairement. Ces condensations étroites apparaissent principalement sur ondes centimétriques et pendant les périodes de forte activité du soleil, et c'est dans ces régions que prennent souvent naissance les "sursauts" observés sur ondes centimétriques. Leur durée de vie paraît plus courte que celle des condensations diffuses (M. R. Kundu, 43).

Il est possible que les augmentations temporaires (gradual rise and fall) du rayonnement observées parfois sur ondes courtes soient produites par des condensations de même nature mais de durée particulièrement brève; elles sont aussi polarisées circulairement et le diamètre de leur source est inférieur à la minute d'arc (M. R. Kundu, 43).

Les émissions de ces condensations radio peuvent sans doute s'identifier au rayonnement thermique de condensations électroniques dans la couronne dont l'épaisseur optique se trouverait éventuellement accrue par la présence du champ magnétique des taches; il semble en effect que la température apparente de ces condensations radio ne soit jamais supérieure à 2 millions de degrés. Notons enfin qu'il existe une relation remarquablement étroite entre le rayonnement global de ces condensations et le rayonnement ionisant qui produit la couche $\mathbf{E}$ de l'ionosphère.

\section{LES CENTRES $R$}

Ces sources de rayonnement radio émettent essentiellement sur ondes métriques un rayonnement polarisé circulairement parfois très intense et irrégulier (orages radio, noise storms, ou enhanced radiation). On peut considérer ce rayonnement comme composé en proportions extrêmement variées d'un continuum plus ou moins stable et de sursauts très brefs (une fraction de seconde) rayonnés sur une faible largeur de bande (quelques mégacycles); ce sont les sursauts (ou bursts) de type I.

Le rayonnement des centres $R$ débute souvent de façon progressive et les centres persistent en général plusieurs jours dans l'atmosphère solaire mais leur position y varie irrégulièrement et il est la plupart du temps difficile de les rattacher sans ambiguïté aussi bien à un centre d'activité optique qu'à 
une condensation radioélectrique stable. Quand on les observe au bord du disque, sur $169 \mathrm{Mc} / \mathrm{s}$ par exemple, leur altitude apparente peut atteindre plusieurs centaines de milliers de kilomètres. Le diamètre apparent de ces centres varie d'une quinzaine de minutes à de faibles valeurs inférieures à une minute d'arc (Y. Avignon, A. Boischot, et P. Simon, 45; A. Hewish, 52; M. H. Cohen et A. D. Fokker, 49; . Elgarøy, 47; U. J. Alekseev et V. V. Vitkevich, 50; T. de Groot, 46).

Jusqu'à présent, aucune théorie satisfaisante n'a été proposée pour expliquer la nature de ces centres et de leur rayonnement. Dans quelques cas, on a mis en évidence un déplacement systématique des fréquences sur lesquelles apparaissent les sursauts, ce qui indique qu'ils peuvent être le siège de mouvements ascendants ou descendants de vitesse voisine de $100 \mathrm{~km} / \mathrm{seconde}$.

Il est possible qu'ils soient plus ou moins reliés à l'activité éruptive des taches mais les recherches effectuées dans ce sens ne paraissent pas encore concluantes.

$$
\text { LES SOURCES TRANSITOIRES ("BURSTS" ET “OUTBURSTS") }
$$

Les émissions de ces sources se caractérisent principalement par la largeur de leur spectre et ses variations en fonction du temps. Les spectrogrammes dynamiques de Wild en donnent la meilleure représentation. On peut distinguer au moins trois types de sources transitoires bien identifiees: celles qui produisent les sursauts de types II, III, et IV. Les deux premiers types, de spectre assez étroit, présentent un glissement caractéristique de la fréquence du maximum d'émission vers les grandes longueurs d'onde en général, et ce glissement est interprété comme l'indication que les sources sont animées de mouvements ascendants dans l'atmosphère solaire; on suppose en effet que leur fréquence d'émission est liée de quelque façon à une fréquence de résonnance de l'atmosphère, soit $f_{c}$, qui dépend de la densité électronique, soit

$$
f_{e}=\frac{1}{2}\left(f_{H}+\sqrt{f_{H}^{2}+4 f_{c}^{2}}\right) \text {, }
$$

qui dépend aussi du champ magnétique; or on doit prévoir une décroissance régulière de $f_{0}$ et $f_{e}$ avec l'altitude; la correspondance qui existe entre ces differentes grandeurs pour des conditions typiques est indiquée dans le Tableau I. Notons qu'une émission synchrotron pourrait aussi présenter un glissement de fréquence fonction de la décroissance du champ magnétique avec l'altitude.

$$
\text { SURSAUTS DU TYPE III ("ISOlated BURSTS," SURSAUTS ISOLES) }
$$

Le rayonnement de ces sursauts (J. P. Wild, K. V. Sheridan et G. H. Trent, 32; A. R. Thompson, 39; F. T. Haddock, 34; C. S. Warwick et J. W. Warwick, 37) couvre une faible largeur de bande (quelques dixièmes de $\mathrm{Mc} / \mathrm{s}$ ). Sa polarisation est variable; l'émission sur la fréquence harmonique apparaît souvent en même temps que le fondamental.

Le glissement en fréquence du maximum d'émission correspond la plupart du temps à des vitesses d'ascension très rapides de l'ordre d'une fraction de 
la vitesse de la lumière. Pourtant certains sursauts qui semblent de nature très voisine, correspondent au contraire à des mouvements descendants (inverted bursts) ou aux deux mouvements successivement ( $U$ bursts) avec des vitesses du même ordre.

Ces sursauts se présentent fréquemment en groupes et le plus souvent à l'occasion d'éruptions chromosphériques. Toutefois, on en a observé certaines séquences qui duraient plusieurs heures ou même plusieurs jours.

Jusqu'à présent et en raison de leur brièveté même on ne possède que fort peu de renseignements sur la structure des sources des types III; seul Wild a pu mesurer leur diamètre, de l'ordre de 10 minutes d'arc sur ondes longues.

La nature de ces sursauts pose deux questions importantes: (1) quelle est la perturbation qui se propage avec une vitesse si élevée: ondes? particules de hautes énergies? et (2) quel est le mécanisme de l'émission: synchrotron? oscillations de plasma?

\section{SURSAUTS DE TYPE II (“OUTBURSTS," GRANDS SURSAUTS)}

Le rayonnement de ces sursauts (J.A. Roberts, 35) couvre une largeur de bande plus grande que celle des précédents; il est faiblement polarisé en général et dure plusieurs minutes. Le glissement en fréquence de ce type de sursauts correspond toujours à un mouvement ascendant dont la vitesse est de l'ordre de quelques centaines de kilometres par seconde.

La structure de l'enregistrement panoramique est toujours extrêmement complexe: on observe d'abord très fréquemment un harmonique en plus du rayonnement sur la fréquence fondamentale. Chacune de ces émissions apparaît souvent elle-même dédoublée (structure à rapprocher des sursauts doubles observés sur ondes centimétriques) et constituée d'une multiplicité de sursauts brefs qu'on a pu comparer à des types III limités à un domaine de fréquence restreint.

Ces sursauts relativement rares et isolés se produisent toujours avec des éruptions chromosphériques. Il paraît certain qu'ils sont associés à une perturbation supersonique issue des basses couches de l'atmosphère solaire.

SURSAUtS DE TYPE IV (“STORM PHASE BURSTS (?), LATE PART"; TYPE 9 DU CRPL)

Le rayonnement de ces sursauts (A. Boischot, 33; J. P. Wild, K. V. Sheridan et G. H. Trent, 32; F. T. Haddock, 34) souvent polarisé circulairement se présente comme un continuum dont le spectre peut couvrir plusieurs octaves. Il est remarquablement stable et dépourvu de sursauts brefs de type I.

Ces sursauts se produisent rarement et tou jours après une éruption chromosphérique importante, parfois 10 à 20 minutes après le début de l'éruption et du sursaut de type II qui les précèdent également; leur durée peut atteindre plusieurs heures.

Les sources de ces sursauts sont d'abord en mouvement ( $V=100$ à 1,000 $\mathrm{km} / \mathrm{seconde}$ ) puis se stabilisent et s'éteignent sur place parfois à plusieurs rayons solaires d'altitude quand on les observe sur ondes métriques.

Il est difficile d'interpréter ces émissions en fonction d'oscillations de plasma, 
TABLEAU I

\begin{tabular}{|c|c|c|c|c|c|}
\hline \multirow{2}{*}{$\begin{array}{l}\text { Sources } \\
\text { d'émission }\end{array}$} & \multicolumn{2}{|c|}{ Caractères de la Source } & \multirow[b]{2}{*}{ Rayonnement } & \multicolumn{2}{|r|}{ Caractères } \\
\hline & Durée & Diamètre & & $\mid \begin{array}{c}\text { Temp.app. } \\
{ }^{\max .} \text { en } \\
{ }^{\circ} \mathrm{K}\end{array}$ & Variabilité \\
\hline $\begin{array}{l}\text { atmosphère } \\
\text { solaire non } \\
\text { perturbée }\end{array}$ & \begin{tabular}{|} 
variation \\
undécennale \\
(?)
\end{tabular} & $\begin{array}{l}\text { >à } 32^{\prime}, \text { croît avec } \\
\lambda \text {; limbe brillant } \\
\text { sur } \mathrm{cm} \text { et dm, } \\
\text { aplatissement } \\
\text { polaire }\end{array}$ & $\begin{array}{l}\text { rayonnement } \\
\text { du soleil } \\
\text { calme }\end{array}$ & $\begin{array}{l}<10^{\circ} \\
\text { croit } \\
\text { avec } \lambda\end{array}$ & stable \\
\hline \multirow{2}{*}{$\begin{array}{l}\text { (condensations } \\
\text { radio) }\end{array}$} & $\begin{array}{l}\text { plusieurs } \\
\text { rotations }\end{array}$ & $\begin{array}{l}1 \text { à } 10^{\prime} \text {, celui } \\
\text { des facules }\end{array}$ & \multirow{2}{*}{$\begin{array}{l}\text { composante } \\
\text { lentement } \\
\text { variable } \\
\text { (slowly vary- } \\
\text { ing compo- } \\
\text { nent) }\end{array}$} & $<2 \times 10^{\circ}$ & stable \\
\hline & $\begin{array}{l}\text { quelques } \\
\text { jours ou } \\
\text { moins }\end{array}$ & $\sim 1^{\prime}$ & & $<5 \times 10^{5}$ & stable \\
\hline $\begin{array}{l}\text { (micro- } \\
\text { condensations) }\end{array}$ & $\begin{array}{l}\text { quelques } \\
\text { minutes } \\
\text { à quelques } \\
\text { heures }\end{array}$ & $\sim \frac{1}{2}^{\prime}$ & $\begin{array}{l}\text { gradual rise } \\
\text { and fall }\end{array}$ & $<5 \times 10^{5}$ & stable \\
\hline (centres $R$ ) & $\begin{array}{l}\text { quelques } \\
\text { heures à } \\
\text { quelques } \\
\text { jours }\end{array}$ & $<1^{\prime}$ à $\sim 10^{\prime}$ & $\begin{array}{l}\text { orage de } \\
\text { bruit, type I } \\
\text { storm }\end{array}$ & $10^{9}$ & $\begin{array}{l}\text { continuum }+ \\
\text { type I en } \\
\text { proportion } \\
\text { variable }\end{array}$ \\
\hline type I & $\begin{array}{l}\text { quelques } \\
\text { dixièmes } \\
\text { de seconde }\end{array}$ & $?$ & type I & $?$ & simple \\
\hline type III & $\begin{array}{l}\text { quelques } \\
\text { secondes }\end{array}$ & $\begin{array}{l}\text { jusqu'à } \sim 10^{\prime}, \\
\text { croît avec } \lambda\end{array}$ & $\begin{array}{l}\text { type III } \\
\text { U, inverted } \\
\text { bursts, fast, } \\
\text { isolated } \\
\text { bursts }\end{array}$ & $>10^{11}$ & $\begin{array}{l}\text { simple ou } \\
\text { en groupes, } \\
\text { harmoniques }\end{array}$ \\
\hline type II & $\begin{array}{l}\text { quelques } \\
\text { minutes }\end{array}$ & $?$ & $\begin{array}{l}\text { type II } \\
\text { outbursts ou } \\
\text { slow bursts }\end{array}$ & $>10^{10}$ & $\begin{array}{l}\text { très } \\
\text { complexe, } \\
\text { harmoniques, } \\
\text { dédoublement }\end{array}$ \\
\hline type IV & $\begin{array}{l}\text { plusieurs } \\
\text { minutes à } \\
\text { plusieurs } \\
\text { heures }\end{array}$ & $\begin{array}{l}\text { jusqu'à } 10^{\prime}, \\
\text { croît avec } \lambda\end{array}$ & $\begin{array}{l}\text { type IV } \\
\text { type } 9 \\
\text { late (storm } \\
\text { phase) burst }\end{array}$ & $10^{11}$ & très stable \\
\hline $\begin{array}{l}\text { sursauts } \\
\text { centimétriques }\end{array}$ & minute & $\sim 2^{\prime}$ & $\begin{array}{l}\text { sursauts } \\
\text { centimétriques }\end{array}$ & $10^{9}$ & $\begin{array}{l}\text { simple + } \\
\text { postburst }\end{array}$ \\
\hline
\end{tabular}


TABLEAU I

\begin{tabular}{|c|c|c|c|c|}
\hline \multicolumn{3}{|l|}{ du Rayonnement } & \multirow{2}{*}{$\begin{array}{l}\text { Phénomènes } \\
\text { associés }\end{array}$} & \multirow{2}{*}{ Remarques } \\
\hline Spectre & Polarisation & Origine & & \\
\hline$\underset{\text { bruit }}{\mathrm{cm}, \mathrm{dm}}, \mathrm{m}$ & non polarisé & thermique & $\begin{array}{l}\text { rayonnement free- } \\
\text { free d'hydrogène } \\
\text { et helium ionisé }\end{array}$ & \\
\hline $\mathrm{cm}, \mathrm{dm}, \mathrm{m}(?)$ & non polarisé & $\begin{array}{l}\text { thermique } \\
(?)\end{array}$ & $\begin{array}{l}\text { condensations } \\
\text { coronales, centres } \\
\text { d'activité optiques, } \\
\text { facules }\end{array}$ & \multirow{3}{*}{$\begin{array}{l}\text { peut être de même } \\
\text { nature }\end{array}$} \\
\hline $\mathrm{cm}, \mathrm{dm}(?)$ & $\begin{array}{l}\text { partiellement } \\
\text { polarisé } \\
\text { circulairement }\end{array}$ & $\begin{array}{c}\text { thermique } \\
(?)\end{array}$ & $\begin{array}{l}+ \text { champ magné- } \\
\text { tique au-dessus } \\
\text { des taches }\end{array}$ & \\
\hline $\mathrm{cm}, \mathrm{dm}$ & $\begin{array}{l}\text { partiellement } \\
\text { polarisé } \\
\text { circulairement }\end{array}$ & $\begin{array}{l}\text { thermique } \\
(?)\end{array}$ & & \\
\hline $\mathrm{m}$ & $\begin{array}{l}\text { polarisé } \\
\text { circulairement }\end{array}$ & $\begin{array}{l}\text { non } \\
\text { thermique } \\
(?)\end{array}$ & $\begin{array}{l}\text { association vague } \\
\text { avec les centres } \\
\text { d'activité optique }\end{array}$ & $\begin{array}{l}\text { la source est susceptible } \\
\text { de lents déplacements } \\
\text { dans la couronne }\end{array}$ \\
\hline$\stackrel{\mathrm{m}}{\text { quelques } \mathrm{Mc} / \mathrm{s}}$ & $\begin{array}{l}\text { polarisé } \\
\text { circulairement }\end{array}$ & $\begin{array}{l}\text { non } \\
\text { thermique } \\
(?)\end{array}$ & $?$ & $\begin{array}{l}\text { prennent leur origine } \\
\text { dans les centres } R\end{array}$ \\
\hline $\begin{array}{l}\mathrm{m}, \mathrm{dm}, 10 \mathrm{Mc} / \mathrm{s} \\
\text { glissement de } \\
\text { fréquence } \\
\pm 100 \mathrm{Mc} / \mathrm{s} / \mathrm{s}\end{array}$ & variable & $\begin{array}{c}\text { synchrotron } \\
\text { (?) } \\
\text { oscillations } \\
\text { de plasma } \\
\text { (?) }\end{array}$ & $\begin{array}{l}\text { souvent avec } \\
\text { première phase } \\
\text { d'une éruption }\end{array}$ & $\begin{array}{l}\text { le glissement de fréquence } \\
\text { correspond à des vitesses } \\
\text { égales à une fraction de } \\
\text { celle de la lumière (par- } \\
\text { ticules de grande éner- } \\
\text { gie? ondes?) }\end{array}$ \\
\hline $\begin{array}{l}\mathrm{m}, \mathrm{dm}, \mathrm{cm}(?), \\
50 \mathrm{Mc} / \mathrm{s} \\
\text { glissement de } \\
\text { fréquence } \\
-1 \mathrm{Mc} / \mathrm{s} / \mathrm{s}\end{array}$ & $\begin{array}{l}\text { en général } \\
\text { peu polarisé }\end{array}$ & $\begin{array}{l}\text { oscillations } \\
\text { de plasma } \\
\text { (?) } \\
\text { synchrotron } \\
\text { (??) }\end{array}$ & $\begin{array}{l}\text { première phase } \\
\text { des éruptions }\end{array}$ & $\begin{array}{c}\text { perturbation en mouve } \\
\text { ment ascendant: } \\
V \text { - } 100-300 \mathrm{~km} / \mathrm{s} \\
\text { (ondes de choc). Origine } \\
\text { des orages magnétiques } \\
\text { (?) }\end{array}$ \\
\hline $\begin{array}{l}\mathrm{cm}, \mathrm{dm}, \mathrm{m}, \\
\text { plusieurs } \\
\text { octaves }\end{array}$ & \begin{tabular}{|l|} 
souvent \\
polarisé \\
circulairement
\end{tabular} & $\begin{array}{c}\text { synchrotron } \\
\text { (?) }\end{array}$ & $\begin{array}{l}\text { après les grosses } \\
\text { éruptions, parfois } \\
10 \text { à } 20^{\mathrm{m}} \text { après }\end{array}$ & $\begin{array}{l}\text { peut-être associé à la } \\
\text { production de rayons } \\
\text { cosmiques solaires; la } \\
\text { source se déplace d'abord } \\
\text { avec des vitesses de } \\
\text { l'ordre de celle des type } \\
\text { II puis se stabilise }\end{array}$ \\
\hline $\mathrm{cm}, \mathrm{dm}$ & variable & $\begin{array}{c}\text { synchrotron } \\
\text { (??) }\end{array}$ & $\begin{array}{l}\text { liaison très étroite } \\
\text { avec les éruptions } \\
\text { chromosphériques }\end{array}$ & \\
\hline
\end{tabular}


il est plus vraisemblable qu'elles soient dues au rayonnement d'électrons de haute énergie produits à la suite de l'éruption.

Il paraît exister d'autres types d'émission encore assez mal définis qui s'apparentent d'une part au type IV par leur durée et leur liaison plus ou moins directe avec des éruptions et d'autre part aux orages radio par leur spectre limité au domaine des ondes métriques et par la variabilité de leur rayonnement. Un exemple extrêmement remarquable d'une émission de ce genre s'est produit le 4 Novembre 1957 (A. Boischot, A. D. Fokker, et P. Simon, 51).

\section{LES EMISSIONS TRANSITOIRES SUR ONDES CENTIMETRIQUES}

La plupart de nos connaissances sur les sources transitoires (H. Tanaka et T. Kakinuma, 41; N. L. Kaìdanovskiī, D. V. Korol'kov, et N. S. Soboleva, 42; M. R. Kundu, 43) proviennent d'observations panoramiques ou interférométriques effectuées dans le domaine des ondes métriques. A priori les émissions transitoires observées sur ondes centimétriques paraissent de nature différente. Les émissions très brèves de types I et III caractéristiques des ondes métriques n'existent pas dans ce domaine de fréquence, où apparaissent seulement des sursauts dont les durées se comptent en minutes.

A côté des sursauts que nous avons déjà cités: les gradual rise and fall que l'on observe communément et les types IV qui eux sont rares, les sursauts typiques sur ondes centimétriques durent quelques minutes. Parfois ces brefs sursauts sont suivis d'une émission temporaire plus durable et moins intense: les postbursts. Malheureusement, ils n'ont fait l'objet d'aucune observation panoramique.

Leur propriété qui paraît la plus importante est leur association extrêmement étroite avec les éruptions optiques. 